\title{
First ISNS Reference Preparation for Neonatal Screening for thyrotropin, phenylalanine and 17a-hydroxyprogesterone in blood spots
}

\author{
L. H. Elvers • J. G. Loeber • J.-L. Dhondt • M. Fukushi • \\ W. H. Hannon • T. Torresani • D. Webster
}

Received: 12 March 2007 /Submitted in revised form: 8 May 2007 / Accepted: 11 May 2007 / Published online: 14 June 2007

(C) SSIEM and Springer 2007

Online citation: JIMD Short Report \#056 (2007) Online

Summary Background: Neonatal screening for congenital disorders like phenylketonuria (PKU), congenital hypothyroidism $(\mathrm{CH})$ and congenital adrenal hyperplasia $(\mathrm{CAH})$ is generally performed in dried blood spots on filter paper. The analytes of interest for testing for $\mathrm{PKU}, \mathrm{CH}$ and $\mathrm{CAH}$ are phenylalanine,

Communicating editor: Rodney Pollitt

\author{
L. H. Elvers $(\bowtie) \cdot$ J. G. Loeber \\ Diagnostic Laboratory for Infectious Diseases \\ and Perinatal Screening, National Institute for Public Health \\ and the Environment, PO Box 1, 3720 BA, \\ Bilthoven, The Netherlands \\ e-mail: bert.elvers@rivm.nl \\ J.-L. Dhondt \\ Hospital Saint Philibert, Lomme Cedex, France \\ M. Fukushi \\ Sapporo City Institute of Public Health, \\ Shiroishi-KU, Sapporo, Japan \\ W. H. Hannon \\ Centers for Disease Control and Prevention, \\ Newborn Screening Branch, Atlanta, Georgia, USA \\ T. Torresani \\ Universitätskinderklinik, Zürich, Switzerland \\ D. Webster \\ National Testing Center, Auckland AK1, New Zealand
}

Electronic Supplementary Material

The online version of this article (doi:10.1007/s10545-007-0622-y)

contains supplementary material, which is available to

authorized users. thyrotropin (TSH) and 17 $\alpha$-hydroxyprogesterone (17OHP), respectively. The International Society for Neonatal Screening (ISNS) decided to prepare a combined reference preparation for the three analytes on filter paper Schleicher \& Schuell \#903, Whatman BFC180 and Toyo Roshi 545. This 'First ISNS Reference Preparation for Neonatal Screening for TSH, phenylalanine and 17OHP in blood spots' (1st ISNSRPNS) has been prepared by the RIVM (Bilthoven). Method: The number of filter paper cards prepared, each with two sets of six blood spot calibrators, was 480, 42 and 69 for Schleicher \& Schuell \#903, Whatman BFC180 and Toyo Roshi 545, respectively. The volume of blood dispensed was $50 \mu$ l. The range of concentrations for TSH was 1-121 mIU/L blood, for phenylalanine $65-865 \mu \mathrm{mol} / \mathrm{L}$ blood and for $17 \mathrm{OHP}$ 2.2-302 nmol/L blood. Results: The linearity of the blood spot calibrators and the homogeneity of the batch (only tested for Schleicher \& Schuell) were good. The differences between the three filter papers were small: i.e. the potency of the ISNS-RPNS on Whatman and Toyo Roshi in terms of Schleicher \& Schuell was between 0.98 and 1.09 for the three analytes. Conclusion: The 1st ISNS-RPNS for TSH, phenylalanine and 17OHP can be said to be suitable as formal reference preparation and as a source for (re)calibrating kit calibrators. 